\title{
Riemann surfaces and Schrödinger potentials of gauged supergravity
}

\author{
I. Bakas, A. Brandhuber, K. Sfetsos* \\ Department of Physics, University of Patras, GR-26500 Patras, Greece \\ Theory Division, CERN, CH-1211 Geneva 23, Switzerland \\ Institut de Physique, Université de Neuchâtel, Breguet 1, CH-2000 Neuchâtel, Switzerland _. . \\ E-mail: bakas@ajax.physics.upatras.gr, brandhu@mail cern.ch, sfetsos@mail.cern.ch
}

ABstract: Supersymmetric domain-wall solutions of maximal gauged supergravity are classified in 4,5 and 7 dimensions in the presence of non-trivial scalar fields taking values in the coset $S L(N, R) / S O(N)$ for $N=8,6$ and 5 respectively. We use an algebro-geometric method based on the Christoffel-Schwarz transformation, which allows for the characterization of the solutions in terms of Riemann surfaces whose genus depends on the isometry group. The uniformization of the curves can be carried out explicitly for models of low genus and results into trigonometric and elliptic solutions for the scalar fields and the conformal factor of the metric. The Schrödinger potentials for the quantum fluctuations of the graviton and scalar fields are derived on these backgrounds and enjoy all properties of supersymmetric quantum mechanics. Special attention is given to a class of elliptic models whose quantum fluctuations are commonly described by the generalized Lamé potential $\mu(\mu+1) \mathcal{P}(z)+\nu(\nu+1) \mathcal{P}\left(z+\omega_{1}\right)+\kappa(\kappa+1) \mathcal{P}\left(z+\omega_{2}\right)+\lambda(\lambda+1) \mathcal{P}\left(z+\omega_{1}+\omega_{2}\right)$ for the Weierstrass function $\mathcal{P}(z)$ of the underlying Riemann surfaces with periods $2 \omega_{1}$ and $2 \omega_{2}$, and for different half-integer values of the coupling constants $\mu, \nu, \kappa, \lambda$.

Recently there has been some progress towards the construction of supersymmetric domainwall solutions of $D$-dimensional gravity coupled to scalar fields taking values in the coset space $S L(N, R) / S O(N)$. There are three cases of particular interest in the context of maximally gauged supergravities, namely $(D, N)=(4,8),(5,6)$ and $(7,5)$. In this contribution we summarize the results obtained in the subject by reducing the classification and construction of all such domain wall solutions to a problem of algebraic geometry, where Riemann surfaces arise naturally in connection with the Christoffel-Schwarz transformation in complex analysis. In the generic case, where no continuous subgroup of the original $S O(N)$ gauge symmetry remains unbroken, we find that the algebraic curve of the corresponding solution is a Riemann surface of genus $N+1$ depending on $N$ real moduli. When some

\footnotetext{
*based on two talks presented by I.B. and K.S.
}

cycles shrink to zero size, by letting some of the moduli coalesce, the symmetry group is enhanced, whereas the genus of the underlying Riemann surface is lowered accordingly. It is then appropriate to think of the breaking of $S O(N)$ to its various subgroups, which remain and characterize the individual solutions, as in the case of spontaneous symmetry breaking. The explicit construction of the domain-walls amounts to the uniformization of the algebraic curves, which can be easily carried out in the cases of low genus ( 0 or 1); as a result, the simplest solutions can be described in terms of elementary functions (rational or trigonometric) for genus 0 surfaces or in terms of elliptic functions for genus 1 surfaces.

A preliminary version of these results was announced last September in the TMR meeting on "Quantum Aspects of Gauge Theories, Supersymmetry and Unification" held in Paris, but a more extensive account was subsequently given 
in two recent papers find more details and a list of references to other work on this interesting topic of research. There are alternative ways to describe our solutions by lifting them to the string/M-theory frame, thus showing that they are consistent truncations of ten- or eleven-dimensional supergravity. In particular, from an eleven-dimensional point of view, the domain-wall solutions of $D=4$ and $D=7$ gauged supergravities correspond to various continuous distributions of M2- and M5-branes respectively. Likewise, from the point of view of ten-dimensional type-IIB supergravity, the domain wall solutions of $D=5$ gauged supergravity correspond to the gravitational field of D3-branes continuously distributed on hyper-surfaces embedded in the six-dimensional space transverse to the branes. Such higher dimensional backgrounds arise in the context of the AdS/CFT correspondence as supergravity duals of the field theories living on M2, M5 or D3 branes on the Coulomb branch, and therefore they have numerous applications. Domain-wall solutions of gauged supergravities are also interesting to consider in relation to proposals that view our world as a membrane embedded non-trivially in a higher dimensional space-time, and for which there is a normalizable graviton zero mode, like in the recent scenario of Randall and Sundrum where a resolution of the mass hierarchy problem in geometrical terms was proposed.

In the present work we will focus mostly on the systematic description of the scalar and graviton field fluctuations on the domain-wall backgrounds, apart from reviewing the general algebrogeometric aspects of the corresponding solutions in gauged supergravities for $D=4,5$ and 7. It turns out that the spectrum of these quantum fluctuations can be formulated as a simple problem in one-dimensional supersymmetric quantum mechanics, where the Schrödinger potential is written in terms of a prepotential given (up to a scale) by the conformal factor of the underlying domain-wall metric. However, the exact form of the spectrum is very difficult to obtain beyond the WKB approximation, in particular for those solutions that correspond to genus 1 (or higher) Riemann surfaces. Nevertheless, in many cases corresponding to genus 0 Riemann surfaces, the exact spectrum can be computed explicitly. We will discuss some aspects of the Schrödinger potentials that arise in several elliptic models, and which have the form of generalized Lamé potentials with half-integer characteristics. Thus, finite-zone potentials, which are familiar from the study of the $\mathrm{KdV}$ equation and the hierarchies of non-linear differential equations descending from it, do not arise in theories of gauged supergravity and as a result there is only very little known now about the exact spectrum. Of course, one might construct domain-wall solutions of $D$-dimensional gravity coupled to a selection of scalar fields outside the scope of gauged supergravities, which could yield finite-zone potentials, but these models would not arise as consistent truncations of ten- or elevendimensional supergravity. These issues pose many interesting questions that deserve further study.

We consider the bosonic sector of gauged supergravity in $D$-dimensions, which contains only scalar fields in the coset $S L(N, R) / S O(N)$, for the specific values of $D$ and $N$ that arise in consistent truncations of ten- or eleven-dimensional supergravity. Since all other fields are set to zero, the Lagrangian assumes the form

$$
\mathcal{L}=\frac{1}{4} \mathcal{R}-\frac{1}{2} \sum_{I=1}^{N-1}\left(\partial \alpha_{I}\right)^{2}-P\left(\alpha_{I}\right)
$$

where the potential $P\left(\alpha_{I}\right)$ has the special form

$$
P\left(\alpha_{I}\right)=\frac{g^{2}}{8}\left(\sum_{I=1}^{N-1}\left(\frac{\partial W}{\partial \alpha_{I}}\right)^{2}-2 \frac{D-1}{D-2} W^{2}\right) \text {. }
$$

The function $W$ is more easily described in terms of $N$ real scalar fields $\beta_{i}$, which are constrained to satisfy the relation

$$
\beta_{1}+\beta_{2}+\cdots+\beta_{N}=0,
$$

as follows

$$
W=-\frac{1}{4} \sum_{i=1}^{N} e^{2 \beta_{i}} .
$$

The precise relation among the fields $\alpha_{I}$ and $\beta_{i}$ is given by

$$
\beta_{i}=\sum_{I=1}^{N-1} \lambda_{i I} \alpha_{I}
$$

where $\lambda_{i I}$ are the elements of an $N \times(N-1)$ matrix. Its rows correspond to the $N$ weights of 
the fundamental representation of $S L(N)$, and as such they satisfy the normalization conditions

$$
\begin{aligned}
\sum_{I=1}^{N-1} \lambda_{i I} \lambda_{j I} & =2 \delta_{i j}-\frac{2}{N}, \quad \sum_{i=1}^{N} \lambda_{i I} \lambda_{i J}=2 \delta_{I J} \\
\sum_{i=1}^{N} \lambda_{i I} & =0 .
\end{aligned}
$$

The coefficient $g^{2}$ appearing in front of the potential $P\left(\alpha_{I}\right)$ defines an associated length scale $R$ given by the relation $g=2 / R$. Having said this we will set $g=1$ in the following.

The domain-wall solutions exhibit $(D-1)$ dimensional Poincaré invariance, namely

$$
\begin{aligned}
d s^{2} & =e^{2 A(z)}\left(\eta_{\mu \nu} d x^{\mu} d x^{\nu}+d z^{2}\right) \\
& =e^{2 A(r)} \eta_{\mu \nu} d x^{\mu} d x^{\nu}+d r^{2}
\end{aligned}
$$

where the metric is given in terms of a single function $A$ which depends on the variable $z$ or $r$, as denoted above; these coordinates are related by the differential relation $d r=-e^{A} d z$. Furthermore, we assume that the scalars depend only on $z$ (or $r$ ) as well. A careful analysis of the problem shows that supersymmetric solutions of this kind satisfy a system of first-order differential equations

$$
\frac{d A}{d r}=-\frac{1}{D-2} W, \quad \frac{d \alpha_{I}}{d r}=\frac{1}{2} \frac{\partial W}{\partial \alpha_{I}} .
$$

These can be obtained either directly from the Killing spinor equations or as a saddle point of the action functional by the method of Bogomol'nyi. In any case, it is convenient to work with the unconstrained fields $\beta_{i}$ as functions of $z$, in terms of which the first-order equations become

$$
\begin{aligned}
A^{\prime} & =\frac{1}{D-2} e^{A} W, \quad \beta_{i}^{\prime}=2 \frac{D-2}{N} A^{\prime}+\frac{1}{2} e^{2 \beta_{i}+A} \\
i & =1,2, \ldots, N,
\end{aligned}
$$

where the prime denotes derivative with respect to $z$.

It is fairly easy to integrate the differential equations for the scalar fields $\beta_{i}$ by introducing an auxiliary function $F(z)$, which is related to the conformal factor as

$$
e^{A(z)}=\left(-F^{\prime}\right)^{\frac{N}{4(D-2)+N}} .
$$

Then, by simple integration, we obtain the following result for the scalar fields,

$$
e^{2 \beta_{i}(z)}=\frac{\left(-F^{\prime}\right)^{\Delta / N}}{F-b_{i}} ; \quad i=1,2, \cdots, N,
$$

where $\Delta=4(D-2) N /(4(D-2)+N)$ and $b_{i}$ are integration constants. Actually, we have $\Delta=4$ for all three cases of interest in gauged supergravity, namely $(D, N)=(4,8),(5,6)$ and $(7,5)$. Moreover, reality of the scalar fields $\beta_{i}$ requires that for real $z$ we have $F(z) \geq b_{\max }$, where $b_{\max }$ is the maximum value of the real moduli $b_{i}$; they may be ordered as $b_{1} \geq b_{2} \geq \cdots \geq b_{N}$ without loss of generality. Taking now into account the algebraic constraint (3) imposed on the scalar fields $\beta_{i}$ we arrive at the following non-linear differential equation for the unknown function $F(z)$

$$
\left(F^{\prime}(z)\right)^{4}=\prod_{i=1}^{N}\left(F(z)-b_{i}\right)
$$

that captures the non-linear aspects of the corresponding equations for the domain-walls for $N=$ 8,6 or 5 . In fact, we will look for solutions which are asymptotic to $A d S_{D}$ space with radius $4(D-2) / N$ (in units where $g=2 / R=1$ ) as $F \rightarrow+\infty$ (or equivalently $z \rightarrow 0^{+}$), when the moduli $b_{i}$ assume arbitrary values. In the special case that all moduli are equal, the corresponding solution is simply $A d S_{D}$ space (not only asymptotically) with all real scalar fields being zero. Hence, classifying the solutions of equation (12), which depend on $N$ real moduli, will provide us with the list of all supersymmetric domain-wall solutions in question. As it turns out, this problem can be addressed systematically in the context of algebraic geometry.

The underlying mathematical structure for solving the differential equation (12), with arbitrary moduli $b_{i}$, is that of the Christoffel-Schwarz transformation. It is useful to think of the variable $z$ as being complex, whereas $F$ taking values in the complex upper-half plane. Of course, appropriate restrictions have to be made at the end in order to ensure the reality of the variable $z$ and hence the reality of our domain-wall solutions. We will treat the Christoffel-Schwarz transformation in a unified way for all three cases of interest, namely $(D, N)=(4,8)$ (M2-branes), 
$(D, N)=(5,6)$ (D3-branes), and $(D, N)=(7,5)$ (M5-branes), since there is a hierarchy of algebraic curves within this transformation that depends on the isometry groups used for the distributions of branes in ten or eleven dimensions. It is useful to start with $N=8$ and consider an octagon in the complex $z$-plane, which is mapped onto the upper-half plane via a Christoffel-Schwarz transformation

$$
\frac{d z}{d F}=\left(F-b_{1}\right)^{-\frac{\varphi_{1}}{\pi}}\left(F-b_{2}\right)^{-\frac{\varphi_{2}}{\pi}} \cdots\left(F-b_{8}\right)^{-\frac{\varphi_{8}}{\pi}} .
$$

This transformation maps the vertices of the octagon to the points $b_{1}, b_{2}, \ldots, b_{8}$ on the real axis of the complex $F$-plane, whereas its interior is mapped onto the entire upper-half $F$-plane. The variables $\varphi_{i}$ denote the exterior (deflection) angles of the octagon at the corresponding vertices, which are constrained by geometry to satisfy the relation $\varphi_{1}+\varphi_{2}+\ldots+\varphi_{8}=2 \pi$. We proceed by making the canonical choice of angles $\varphi_{1}=\varphi_{2}=$ $\ldots=\varphi_{8}=\pi / 4$, in which case we arrive at the differential equation that relates $d z$ and $d F$ :

$$
\left(\frac{d z}{d F}\right)^{4}=\left(F-b_{1}\right)^{-1}\left(F-b_{2}\right)^{-1} \cdots\left(F-b_{8}\right)^{-1}
$$

which is the equation we have to solve for the case of $D=4$ gauged supergravity with scalar fields in the coset $S L(8, R) / S O(8)$.

It is convenient at this point to introduce complex algebraic variables

$$
x=F(z), \quad y=F^{\prime}(z),
$$

which cast the differential equation above into the form of an algebraic curve

$$
y^{4}=\left(x-b_{1}\right)\left(x-b_{2}\right) \cdots\left(x-b_{8}\right) \text {. }
$$

This defines a Riemann surface, which is pictured geometrically by gluing four sheets together along their branch cuts. The task is to uniformize the algebraic curve by finding another complex variable, call it $u$, so that $x=x(u)$ and $y=y(u)$, which resolves the problem of multi-valuedness of the algebraic equation above. Then, following the definition of $x$ and $y$ in terms of $F(z)$ and its $z$-derivative, one can apply the chain rule in order to obtain the function $z(u)$ by integration of the resulting first-order equation

$$
\frac{d z}{d u}=\frac{1}{y(u)} \frac{d x(u)}{d u} .
$$

Finally, by inverting the result one obtains the function $u(z)$, which yields $F(z)$, and hence the conformal factor of the corresponding domain wall solutions, together with the solution for the scalar fields. Of course, there is an integration constant that appears in the function $z(u)$, but this can be fixed by requiring that the asymptotic behaviour of the domain-walls approach the $A d S$ geometry as $z \rightarrow 0^{+}$. We also note that there is a discrete symmetry $x \leftrightarrow-x, b_{i} \leftrightarrow-b_{i}$ that leaves invariant the form of the algebraic curve. It can be employed in order to set $F$ bigger or equal than the maximum value of the moduli $b_{i}$ instead of being smaller or equal than the minimum value, thus insuring that $z \rightarrow 0^{+}$corresponds to $F \rightarrow+\infty$ instead of $-\infty$.

The whole procedure is straightforward, but turns out to be cumbersome when the moduli $b_{i}$ take general values. Matters simplify considerably when certain moduli are allowed to become equal, which effectively reduces the genus of the algebraic curve and leaves some of the isometries unbroken. In general we will have models for each continuous subgroup of the maximal isometry group $S O(8)$, in which case the associated Riemann surface becomes

$$
y^{4}=\left(x-b_{1}\right)^{k_{1}}\left(x-b_{2}\right)^{k_{2}} \cdots\left(x-b_{k}\right)^{k_{r}},
$$

with $k_{1}+k_{2}+\cdots+k_{r}=8$, and $S O\left(k_{1}\right) \times S O\left(k_{2}\right) \times$ $\cdots \times S O\left(k_{r}\right)$ as isometry group. These surfaces will not be in an irreducible form if all the exponents $k_{i}$ have a common divisor with 4 . To calculate the genus, and also proceed with their uniformization, we first bring the algebraic curves into the irreducible form (when this is necessary)

$$
y^{m}=\left(x-b_{1}\right)^{a_{1}}\left(x-b_{2}\right)^{a_{2}} \cdots\left(x-b_{n}\right)^{a_{n}},
$$

where the integer exponents (with $n \leq 8$ ) satisfy the relation $a_{1}+a_{2}+\cdots a_{n}=2 m$. Then, we write down the ratios

$\frac{a_{1}}{m}=\frac{d_{1}}{c_{1}}, \cdots, \frac{a_{n}}{m}=\frac{d_{n}}{c_{n}} ; \quad \frac{a_{1}+\cdots+a_{n}}{m}=\frac{d_{0}}{c_{0}}$ 
in terms of relatively prime numbers and use the Riemann-Hurwitz relation

$$
g=1-m+\frac{m}{2} \sum_{i=0}^{n}\left(1-\frac{1}{c_{i}}\right)
$$

to compute the genus $g$ (it should not be confused with the symbol used for the inverse length scale $g=2 / R$, which has already been normalized to $1)$.

We present below the list of all Riemann surfaces that classify the domain-wall solutions of four-dimensional gauged supergravity with nontrivial scalar fields in the coset $S L(8, R) / S O(8)$ by giving their genus according to the RiemannHurwitz relation, their irreducible form (since in certain cases the exponents have common factors and the curve might be reducible when written in its original form), as well as the corresponding isometry groups that determine the geometrical distribution of M2-branes in eleven dimensions. We have 22 models in total, namely:

$\underline{g=9}$

$\frac{g=9}{y^{4}=}\left(x-b_{1}\right)\left(x-b_{2}\right) \cdots\left(x-b_{7}\right)\left(x-b_{8}\right)$ having no isometry group.

$\frac{g=7}{y^{4}=}\left(x-b_{1}\right)\left(x-b_{2}\right) \cdots\left(x-b_{6}\right)\left(x-b_{7}\right)^{2}$ with isometry group $S O(2)$.

$\frac{g=6}{y^{4}=}\left(x-b_{1}\right)\left(x-b_{2}\right) \cdots\left(x-b_{5}\right)\left(x-b_{6}\right)^{3}$ with isometry group $S O(3)$.

$\underline{g=5}$

$\overline{y^{4}=}\left(x-b_{1}\right) \cdots\left(x-b_{4}\right)\left(x-b_{5}\right)^{2}\left(x-b_{6}\right)^{2}$ with isometry group $S O(2)^{2}$.

$\underline{g=4}$

$\frac{\overline{y^{4}}=}{y^{2}}\left(x-b_{1}\right)\left(x-b_{2}\right)\left(x-b_{3}\right)\left(x-b_{4}\right)^{2}\left(x-b_{5}\right)^{3}$ with isometry group $S O(2) \times S O(3)$.

$\underline{g=3}$

$\overline{y^{4}=}\left(x-b_{1}\right) \cdots\left(x-b_{4}\right)\left(x-b_{5}\right)^{4}$ with isometry group $S O(4)$;

$y^{4}=\left(x-b_{1}\right)\left(x-b_{2}\right)\left(x-b_{3}\right)\left(x-b_{4}\right)^{5}$ with isometry group $S O(5)$;

$y^{4}=\left(x-b_{1}\right)\left(x-b_{2}\right)\left(x-b_{3}\right)^{3}\left(x-b_{4}\right)^{3}$ with isometry group $S O(3)^{2}$;

$y^{4}=\left(x-b_{1}\right)\left(x-b_{2}\right)\left(x-b_{3}\right)^{2}\left(x-b_{4}\right)^{2}\left(x-b_{5}\right)^{2}$ with isometry group $S O(2)^{3}$.

$\frac{g=2}{y^{4}=}\left(x-b_{1}\right)\left(x-b_{2}\right)^{2}\left(x-b_{3}\right)^{2}\left(x-b_{4}\right)^{3}$ with isometry group $S O(2)^{2} \times S O(3)$. $\underline{g=1}$

$\frac{\bar{y} y^{4}=}{}\left(x-b_{1}\right)\left(x-b_{2}\right)\left(x-b_{3}\right)^{6}$ with isometry group $S O(6)$;

$y^{4}=\left(x-b_{1}\right)\left(x-b_{2}\right)\left(x-b_{3}\right)^{2}\left(x-b_{4}\right)^{4}$ with isometry group $S O(2) \times S O(4)$;

$y^{4}=\left(x-b_{1}\right)\left(x-b_{2}\right)^{2}\left(x-b_{3}\right)^{5}$ with isometry group $S O(2) \times S O(5)$;

$y^{4}=\left(x-b_{1}\right)^{2}\left(x-b_{2}\right)^{3}\left(x-b_{3}\right)^{3}$ with isometry group $S O(2) \times S O(3)^{2}$;

$y^{2}=\left(x-b_{1}\right)\left(x-b_{2}\right)\left(x-b_{3}\right)\left(x-b_{4}\right)$ with isometry group $S O(2)^{4}$.

$\underline{g=0}$

$\frac{g=0}{y^{4}=}\left(x-b_{1}\right)\left(x-b_{2}\right)^{7}$ with isometry group $S O(7)$;

$y=(x-b)^{2}$ with maximal isometry $S O(8)$;

$y^{2}=\left(x-b_{1}\right)\left(x-b_{2}\right)^{3}$ with isometry group $S O(2) \times$ $S O(6)$;

$y^{4}=\left(x-b_{1}\right)\left(x-b_{2}\right)^{3}\left(x-b_{3}\right)^{4}$ with isometry group $S O(3) \times S O(4)$;

$y^{4}=\left(x-b_{1}\right)^{3}\left(x-b_{2}\right)^{5}$ with isometry group $S O(3) \times S O(5)$

$y=\left(x-b_{1}\right)\left(x-b_{2}\right)$ with isometry group $S O(4)^{2}$; $y^{2}=\left(x-b_{1}\right)\left(x-b_{2}\right)\left(x-b_{3}\right)^{2}$ with isometry group $S O(2)^{2} \times S O(4)$.

It is interesting to note that the classification of domain-walls of five-dimensional gauged supergravity with non-trivial scalar fields in the coset $S L(6, R) / S O(6)$ follows immediately from above by restricting our attention to models with an $S O(2)$ factor in the isometry group. It is clear that in this case the classification of solutions reduces to the list of all algebraic curves

$$
y^{4}=\left(x-b_{1}\right)\left(x-b_{2}\right) \cdots\left(x-b_{6}\right),
$$

depending on the values of the six real moduli $b_{i}$. But such curves can be viewed as special cases of the $N=8$ curves when $b_{7}=b_{8}=$ $-\infty$; the limiting point is taken to be $-\infty$ rather than $+\infty$ in order to keep the ordering $b_{1} \geq$ $b_{2} \geq \cdots \geq b_{8}$ that is usually made. In other words, using the geometrical framework of the Christoffel-Schwarz transformation, we consider that the octagon in the complex $z$-plane degenerates by shrinking one of its sides to zero size (in which case the corresponding deflection angle becomes $\pi / 2$ ) and the resulting double vertex is mapped to $-\infty$ on the real $F$-line. Therefore, by comparison with the list above, we obtain immediately the table of all domain-walls 
of five-dimensional gauged supergravity, which correspond to various continuous distributions of D3-branes in ten dimensions. We have 11 such models in total, as follows by inspection, which maintain the genus of their "parent" $N=8$ algebraic curves; $g$ is ranging now from 7 to 0 depending on the isometry groups of the individual models.

Finally, the algebraic classification of all domain wall solutions of seven-dimensional gauged supergravity with non-trivial scalar fields in the coset $S L(5, R) / S O(5)$ (which by the way provides the full scalar sector in this case) follows by considering all Riemann surfaces of the form

$$
y^{4}=\left(x-b_{1}\right)\left(x-b_{2}\right) \cdots\left(x-b_{5}\right),
$$

for various values of the five real moduli $b_{i}$. As before, these surfaces can be viewed as special cases of the $N=8$ algebraic curves where three of the moduli are taken to infinity, i.e. $b_{6}=b_{7}=$ $b_{8}=-\infty$, whereas the remaining are free to vary. As before, in terms of the Christoffel-Schwarz transformation, the original octagon in the complex $z$-plane degenerates to an exagon with one of its deflection angles becoming now $3 \pi / 4$, and the resulting triple vertex is mapped to $-\infty$ on the real $F$-line. Put differently, we may compose the list of all domain-walls that correspond to various continuous distributions of M5-branes in eleven dimensions by considering all $N=8$ models with a $S O(3)$ isometry factor. Thus, we have 7 such models in total, which follow by inspection from the list above, all having the same genus as their "parent" $N=8$ algebraic curves; $g$ is now ranging from 6 to 0 depending on the isometry group. We note for completeness in this latter case that the invariance of the curves under the discrete symmetry $x \leftrightarrow-x, b_{i} \leftrightarrow-b_{i}$ is not present any more, because the corresponding algebraic equations contain only an odd number of factors.

In summary, for generic values of the moduli parameters $b_{i}$, the domain-wall solutions of $D$ dimensional gauged supergravity are described by a Riemann surface of genus $N+1$ in the presence of non-trivial scalar fields in the coset space $S L(N, R) / S O(N)$. As certain cycles shrink to zero size by letting some of the moduli coalesce, the genus of the algebraic curve becomes smaller and the corresponding domain-wall solutions have as symmetry the appropriate subgroups of $S O(N)$. The explicit construction of the solutions requires to perform the uniformization of the associated Riemann surfaces, which can be easily done for the cases of low genus, namely 0 or 1 . For genus 0 , one has to employ bi-rational transformations from $x, y$ to new variables $v(x, y), w(x, y)$, so that the algebraic curve assumes the unicursal form that can be easily uniformized using a complex variable $u$ as $v=w=u$. Then, the domain-wall solutions can be expressed as rational or trigometric functions in $u$, and hence $z$, when $u(z)$ is invertible in closed form. For genus 1, suitable bi-rational transformations to new variables $v(x, y), w(x, y)$ will cast the curve into its standard Weierstrass form

$$
w^{2}=4 v^{3}-g_{2} v-g_{3},
$$

which can be uniformized using the Weierstrass function $\mathcal{P}(u)$, with complex variable $u$ in the fundamental domain defined by the two periods $2 \omega_{1}$ and $2 \omega_{2}$ of the surface, so that $v=\mathcal{P}(u)$ and $w=\mathcal{P}^{\prime}(u)$. Then, the corresponding domainwall solutions can be described explicitly in terms of elliptic functions, at least when $u(z)$ can be found in closed form. We will present some explicit examples of this type later. For models that correspond to higher genus surfaces, $g \geq 2$, the uniformization is mathematically much more involved and will not be addressed here.

The application that we intend to consider in some detail in the following concerns the spectrum of the quantum fluctuations for the graviton as well as the scalar fields on the domain-wall backgrounds of gauged supergravity, which they turn out to coincide. Using the ansatz

$$
\Phi(x, z)=\exp (i k \cdot x) \exp \left(-\frac{D-2}{2} A\right) \Psi(z)
$$

for a massless scalar field or any of the components of the graviton tensor field, which represents plane waves propagating along the $(D-$ 2)-brane with an amplitude function that is $z$ dependent, we find that the spectrum of fluctuations is described by a one-dimensional quantum mechanical problem. Setting $M^{2}=-k \cdot k$ for the mass-square, we obtain a time-independent 
Schrödinger equation for the function $\Psi(z)$, namely

$$
-\Psi^{\prime \prime}(z)+V(z) \Psi(z)=M^{2} \Psi(z) .
$$

The potential is determined by the conformal factor of the metric of the domain-wall background, $A(z)$, by

$$
V=\frac{(D-2)^{2}}{4} A^{\prime 2}+\frac{D-2}{2} A^{\prime \prime},
$$

which is of the form appearing in problems of supersymmetric quantum mechanics.

The potential $V(z)$ can be determined explicitly once the uniformization of the underlying algebraic curve has been carried out in detail. The form can be rather complicated, depending on the specific models, but for all of them the potential has the asymptotic form

$$
V(z) \simeq \frac{D(D-2)}{4} \frac{1}{z^{2}}, \quad \text { as } z \rightarrow 0^{+},
$$

since the space approaches $A d S_{D}$ in the limit $z \rightarrow 0^{+}$(or equivalently $F \rightarrow+\infty$ ). This means that the potential is unbounded from above. Its behaviour close to the other end, namely $F \rightarrow$ $b_{\max }$, depends on the multiplicity of $b_{\max }$ in the algebraic form of the curve. A careful analysis of the problem shows that if $b_{\text {max }}$ appears $n$ times (and so the model has an isometry group with an $S O(n)$ factor), the potential will behave (including a subscript $n$ to distinguish among various cases) as

$$
\begin{aligned}
& V_{n} \simeq \frac{f_{0}^{1 / 2}}{64}\left(3 n^{2}-8 n\right)\left(F-b_{\max }\right)^{\frac{1}{2} n-2} \\
& \text { as } \quad F \rightarrow b_{\max }
\end{aligned}
$$

where $f_{0}=\prod_{i=n+1}^{N}\left(b_{1}-b_{i}\right)\left(\operatorname{choosing} b_{1} \equiv b_{\max }\right)$ is a constant. Hence, for $n>4$, the potential goes to zero at the other end and consequently the spectrum is continuous. For $n=4$, the potential approaches a constant value, $f_{0}^{1 / 2} / 4$, and so the spectrum is again continuous but there is a mass gap whose squared value is given by $f_{0}^{1 / 2} / 4$. In both cases above, namely when $n \geq 4$, the range of $z$ necessarily extends from 0 to $+\infty$. For $n<4$, on the other hand, the spectrum is discrete and $z$ extends from 0 to some maximum (but finite) value $z_{\max }$, which is determined by the algebraic equation $F\left(z_{\max }\right)=b_{\max }$. In fact we find in this case that

$$
\begin{aligned}
& V_{n} \simeq\left(\frac{(n-2)^{2}}{(n-4)^{2}}-\frac{1}{4}\right) \frac{1}{\left(z-z_{\max }\right)^{2}} \\
& \text { as } \quad z \rightarrow z_{\max }^{-}
\end{aligned}
$$

and the potential goes there to $+\infty$ for $n=3$ and to $-\infty$ for $n=1,2$. The latter two cases are not pathological since the coefficient of the $1 /\left(z-z_{\max }\right)^{2}$ term is larger or equal to $-1 / 4$ as required from elementary quantum mechanical considerations. Equivalently, since the potential has the form (27) appearing in supersymmetric quantum mechanics, the spectrum always has to be bounded from below by zero. This completes the brief qualitative discussion of the spectrum in all cases of interest.

In supersymmetric quantum mechanics there is a superpotential $W(z)$ and a pair of Schrödinger potentials associated to it

$$
V_{-}=W^{2}-W^{\prime}, \quad V_{+}=W^{2}+W^{\prime} .
$$

Their spectrum are closely related to each other, and the same is true for the corresponding eigenfunctions, although there are some technical issues depending on whether supersymmetry is broken or not. In our case the superpotential is provided by the conformal factor of the metric, up to a scale,

$$
W(z)=\frac{D-2}{2} A^{\prime}(z)
$$

and the potential of the Schrödinger equation that describes the quantum fluctuations of the scalar and graviton fields on the domain-wall backgrounds of gauged supergravity (for $D=4,5$ or 7 ) is given by the form $V_{+}$. The partner potential $V_{-}$sometimes turns out to be easier to analyse quantum mechanically, although generically it does not itself correspond to the Schrödinger potential of a domain-wall solution of gauged supergravity. Note at this point that if $A \rightarrow-A$ the superpotential will also flip sign and there will be an interchange $V_{+} \leftrightarrow V_{-}$. However, as $z \rightarrow 0$, the $A d S_{D}$ asymptotic behaviour of the domain-walls is not preserved under this interchange, and hence it can only be of mathematical interest for computing the spectrum using $V_{-}$instead of $V_{+}$. We will return to this later 
with specific examples. Finally, we mention that it is possible to apply the WKB approximation, which works very well in supersymmetric quantum mechanics, in order to get results for the spectrum of quantum fluctuations on domainwall backgrounds. Here, we will focus attention on the possibility to describe the spectrum exactly, in particular for domain-walls associated to genus 1 Riemann surfaces where the Schrödinger potential is expressed via the Weierstrass function in a generalized Lamé form. We will see that unlike other problems in physics, where Lamé potentials with integer characteristics become relevant, the characteristics turn out to be halfinteger in gauged supergravity. Consequently, the spectrum is not of finite-zone type, and hence much more difficult to study exactly. Thus, a number of questions will be left open for future study.

We proceed with the presentation of some explicit examples of elliptic type using the algebraic classification of domain-walls in terms of Riemann surfaces and isometry groups. In all these cases the Schrödinger potentials will assume the common form

$$
\begin{aligned}
V(z) & =\mu(\mu+1) \mathcal{P}(z)+\nu(\nu+1) \mathcal{P}\left(z+\omega_{1}\right) \\
& +\kappa(\kappa+1) \mathcal{P}\left(z+\omega_{2}\right) \\
& +\lambda(\lambda+1) \mathcal{P}\left(z+\omega_{1}+\omega_{2}\right)
\end{aligned}
$$

for different values of the coupling constants $\mu$, $\nu, \kappa, \lambda$ that will be determined in each case separately.

$S O(2) \times S O(2) \times S O(2)$ in $D=5:$ The irreducible form of the algebraic curve is written directly in (hyper)-elliptic form

$$
y^{2}=\left(x-b_{1}\right)\left(x-b_{2}\right)\left(x-b_{3}\right)
$$

and it can be brought into the standard Weierstrass form $w^{2}=4 v^{3}-g_{2} v-g_{3}$ by the simple transformation

$$
y=4 w, \quad x=4 v+\frac{1}{3}\left(b_{1}+b_{2}+b_{3}\right),
$$

in which case we find that

$$
\begin{aligned}
g_{2}=\frac{1}{36} & \left(\left(b_{1}+b_{2}-2 b_{3}\right)^{2}-\left(b_{2}+b_{3}-2 b_{1}\right)\right. \\
& \left.\times\left(b_{1}+b_{3}-2 b_{2}\right)\right) \\
g_{3}=- & \frac{1}{432}\left(b_{1}+b_{2}-2 b_{3}\right)\left(b_{2}+b_{3}-2 b_{1}\right) \\
& \times\left(b_{1}+b_{3}-2 b_{2}\right)
\end{aligned}
$$

Then, performing the uniformization in terms of the Weierstrass function $\mathcal{P}(u)$ of a complex parameter $u$, we find that $z=4 u$. The conformal factor of the metric turns out to be

$$
e^{2 A(z)}=\left(\frac{1}{16} \mathcal{P}^{\prime}(u)\right)^{2 / 3}
$$

and so the corresponding domain-wall solution approaches $A d S_{5}$ (with radius 2) as $z \rightarrow 0^{+}$. On the other hand, since the uniformizing parameter $u$ assumes real values from 0 to $\omega_{1}$ (real semiperiod), we have that $z$ varies from 0 to $4 \omega_{1}$.

In this case, the corresponding potential in the Schrödinger differential equation for the variable $u$ is

$$
\begin{aligned}
V(u) & =\frac{1}{4}\left(15 \mathcal{P}(u)-\mathcal{P}\left(u+\omega_{1}\right)-\mathcal{P}\left(u+\omega_{2}\right)\right. \\
& \left.-\mathcal{P}\left(u+\omega_{1}+\omega_{2}\right)\right)
\end{aligned}
$$

and so it has the generalized Lamé form (33) with half-integer coupling constants $\mu=3 / 2, \nu=\kappa=$ $\lambda=-1 / 2$. Using the identity

$$
\begin{aligned}
4 \mathcal{P}(2 u) & =\mathcal{P}(u)+\mathcal{P}\left(u+\omega_{1}\right)+\mathcal{P}\left(u+\omega_{2}\right) \\
& +\mathcal{P}\left(u+\omega_{1}+\omega_{2}\right)
\end{aligned}
$$

it can be written into another form that we present here together with the partner supersymmetric potential

$$
V_{-}(u)=3 \mathcal{P}(2 u), \quad V_{+}(u)=4 \mathcal{P}(u)-\mathcal{P}(2 u) .
$$

It is interesting to note in this case that the partner potential $V_{-}$defines a simpler Schrödinger problem in the variable $\tilde{u}=2 u$ having $V(\tilde{u})=$ $n(n+1) \mathcal{P}(\tilde{u})$ with $n=1 / 2$.

$S O(3) \times S O(3)$ in $D=5$ : In this case the algebraic curve of the model has the form

$$
y^{4}=\left(x-b_{1}\right)^{3}\left(x-b_{2}\right)^{3},
$$

which can be brought into the Weierstrass form $w^{2}=4 v^{3}-g_{2} v-g_{3}$ using the transformation

$$
\begin{aligned}
& x=b_{1}+\frac{1}{v}\left(v+\frac{1}{4}\left(b_{2}-b_{1}\right)\right)^{2}, \\
& y=\frac{w^{3}}{8 v^{3}},
\end{aligned}
$$

where

$$
g_{2}=\frac{1}{4}\left(b_{1}-b_{2}\right)^{2}, \quad g_{3}=0
$$


Performing the uniformization in terms of the Weierstrass function $\mathcal{P}(u)$ of a complex variable $u$, we find that $z=-8 u$. Moreover, the conformal factor of the domain-wall metric is given by

$$
e^{2 A(z)}=\left(\frac{\mathcal{P}^{\prime}(u)}{8 \mathcal{P}(u)}\right)^{2} .
$$

Clearly, this conformal factor approaches $4 / z^{2}$ as $z \rightarrow 0$, which is the asymptotic $A d S_{5}$ limit of the solution (with radius 2). In this case $z$ ranges from 0 to $8 \omega_{1}$.

The Schrödinger potential for the corresponding differential equation written using the variable $u$ turns out to be

$$
\begin{aligned}
V(u) & =\frac{1}{4}\left(15 \mathcal{P}(u)+3 \mathcal{P}\left(u+\omega_{1}\right)+3 \mathcal{P}\left(u+\omega_{2}\right)\right. \\
& \left.+15 \mathcal{P}\left(u+\omega_{1}+\omega_{2}\right)\right),
\end{aligned}
$$

which also has the generalized Lamé form (33) with half-integer coupling constants $\mu=\lambda=3 / 2$ and $\nu=\kappa=1 / 2$. Its supersymmetric partner can be easily determined and turns out to be of the generalized Lamé form with $\mu=\lambda=1 / 2$ and $\nu=\kappa=3 / 2$. These potentials exhibit a special invariance under $u \rightarrow u+\omega_{1}+\omega_{2}$ due to the $S O(3) \times S O(3)$ symmetry of the underlying domain-wall solution.

$S O(2) \times S O(3)$ in $D=7$ : The genus 1 curve of this model is given by

$$
y^{4}=\left(x-b_{1}\right)^{2}\left(x-b_{2}\right)^{3} .
$$

It can be brought into the standard Weierstrass form $w^{2}=4 v^{3}-g_{2} v-g_{3}$ using the transformation

$$
\begin{aligned}
& x=\frac{b_{2}-b_{1}}{4} \frac{w^{2}}{v^{3}}+b_{1}, \\
& y=\left(b_{2}-b_{1}\right) \frac{w}{v}\left(\frac{w^{2}}{4 v^{3}}-1\right),
\end{aligned}
$$

where it turns out that

$$
g_{2}=\frac{1}{4}\left(b_{1}-b_{2}\right), \quad g_{3}=0 .
$$

We find that the uniformizing parameter $u$ is related to $z$ via

$$
u=\omega_{1}+\omega_{2}-\frac{z}{2}
$$

whereas the conformal factor of the metric turns out to be

$$
e^{2 A(z)}=\left(\frac{\left(b_{1}-b_{2}\right)^{2}}{16} \frac{\mathcal{P}^{\prime}(u)}{\mathcal{P}^{3}(u)}\right)^{2 / 5}
$$

The real variable $z$ ranges from 0 to $2 \omega_{1}$ if $b_{1}>$ $b_{2}$ and from 0 to $2\left(\omega_{1}+\omega_{2}\right)$ if $b_{1}<b_{2}$. The domain-wall solution approaches asymptotically $A d S_{7}$ (with radius 4 ) as $z \rightarrow 0^{+}$.

The Schrödinger potential takes again the generalized Lamé form (33), after rescaling $z$ by a factor of 2 , i.e. $z \rightarrow 2 z$,

$$
\begin{aligned}
V(z) & =\frac{1}{4}\left(35 \mathcal{P}(z)-\mathcal{P}\left(z+\omega_{1}\right)-\mathcal{P}\left(z+\omega_{2}\right)\right. \\
& \left.+3 \mathcal{P}\left(z+\omega_{1}+\omega_{2}\right)\right)
\end{aligned}
$$

with coupling constants $\mu=5 / 2, \nu=\kappa=-1 / 2$, $\lambda=1 / 2$. Its supersymmetric partner is easily determined to be of the generalized Lamé type with coupling constants $\mu=\lambda=3 / 2$ and $\nu=$ $\kappa=1 / 2$, which concides with the Schrödinger potential for the quantum fluctuations on the $S O(3) \times S O(3)$ domain-wall model in $D=5$ gauged supergravity.

$S O(2) \times S O(2) \times S O(2) \times S O(2)$ in $D=4:$ Another notable example is the genus 1 model described by the algebraic curve in (hyper)-elliptic form

$$
y^{2}=\left(x-b_{1}\right)\left(x-b_{2}\right)\left(x-b_{3}\right)\left(x-b_{4}\right) .
$$

Setting $b_{4}=0$ without loss of generality, we employ the transformation

$$
\begin{aligned}
& x=\frac{1}{\frac{1}{3}\left(b_{1}^{-1}+b_{2}^{-1}+b_{3}^{-1}\right)-v} \\
& y=\frac{\sqrt{b_{1} b_{2} b_{3}}}{2} \frac{w}{\left(\frac{1}{3}\left(b_{1}^{-1}+b_{2}^{-1}+b_{3}^{-1}\right)-v\right)^{2}}(5
\end{aligned}
$$

to bring the curve into its standard Weierstrass form $w^{2}=4 v^{3}-g_{2} v-g_{3}$ with

$$
\begin{aligned}
g_{2}= & \frac{2}{9}\left(\left(b_{1}^{-1}+b_{2}^{-1}-2 b_{3}^{-1}\right)^{2}\right. \\
+ & \left(b_{2}^{-1}+b_{3}^{-1}-2 b_{1}^{-1}\right)^{2} \\
+ & \left.\left(b_{3}^{-1}+b_{1}^{-1}-2 b_{2}^{-1}\right)^{2}\right), \\
g_{3}= & \frac{4}{27}\left(b_{1}^{-1}+b_{2}^{-1}-2 b_{3}^{-1}\right)\left(b_{2}^{-1}+b_{3}^{-1}-2 b_{1}^{-1}\right) \\
& \times\left(b_{3}^{-1}+b_{1}^{-1}-2 b_{2}^{-1}\right) .
\end{aligned}
$$

Then, uniformizing the curve, as usual, in terms of the Weierstrass function $\mathcal{P}(u)$ of a complex variable $u$, we find that $z$ is related to it by

$$
\begin{aligned}
u & =\frac{\sqrt{b_{1} b_{2} b_{3}}}{2} z+c ; \quad \text { where } \\
\mathcal{P}(c) & =\frac{1}{3}\left(b_{1}^{-1}+b_{2}^{-1}+b_{3}^{-1}\right) .
\end{aligned}
$$


Also, the conformal factor of the corresponding domain-wall solution turns out to be

$$
e^{2 A(z)}=\frac{b_{1} b_{2} b_{3}}{4}(\mathcal{P}(u-c)-\mathcal{P}(u+c))
$$

which approaches the $A d S_{4}$ limit $1 / z^{2}$ as $z \rightarrow 0^{+}$. It also turns out in this case that $z$ ranges from 0 to a maximum value given by $2\left(\omega_{1}-c\right) / \sqrt{b_{1} b_{2} b_{3}}$.

The Schrödinger potential is calculated to be

$$
V(z)=\frac{b_{1} b_{2} b_{3}}{4}(2 \mathcal{P}(u+c)+2 \mathcal{P}(u-c)-\mathcal{P}(2 u))
$$

and it appears to be different from the generalized Lamé form above. It is instructive, however, to work out the supersymmetric partner potential. We find in this case, when the Schrödinger equation is written in terms of the variable $u$, that the partners are

$$
\begin{aligned}
& V_{-}=3 \mathcal{P}(2 u) \\
& V_{+}=2 \mathcal{P}(u+c)+2 \mathcal{P}(u-c)-\mathcal{P}(2 u)
\end{aligned}
$$

and so by an appropriate scaling of variable, $\tilde{u}=$ $2 u$, the partner Schrödinger problem has again the special Lamé potential $V(\tilde{u})=n(n+1) \mathcal{P}(\tilde{u})$ with $n=1 / 2$. The only difference from the previous case, where the $n=1 / 2$-Lamé potential makes its appearence, is that the variable $\tilde{u}$ ranges from $2 c$ to $2 \omega_{1}$, instead of the interval 0 to $2 \omega_{1}$.

It will be interesting to revisit in future work the spectral properties of the generalized Lamé potentials with half-integer coupling constants, in view of their relevance in theories of gauged supergravity. There is only very little work on this problem, which dates back to last century, and apparently turns out that such potentials are of infinite-zone type. Their structure becomes tractable when the underlying genus 1 Riemann surfaces degenerate by shrinking their $a$ - or $b$ cycles to zero size, in which case the exact spectrum is known and coincides with the spectrum of the quantum Calogero system. In this limit the potential becomes trigonometric and hence the states are given in terms of elementary functions. For the elliptic models that arise here, however, only the results from the WKB approximation are presently known to the authors.

Finally, another interesting problem is the systematic construction of solutions in the sector of gauged supergravity that also contains gauge fields. The methods of algebraic geometry might prove again useful for studying such generalizations.

\section{Acknowledgements}

We are grateful to the organizers of the TMR meeting for their kind invitation and generous financial support.

\section{References}

[1] I. Bakas and K. Sfetsos, States and curves of five-dimensional gauged supergravity, to appear in Nucl. Phys. B, hep-th/9909041.

[2] I. Bakas, A. Brandhuber and K. Sfetsos, Domain walls of gauged supergravity, M-branes, and algebraic curves, hep-th/9912132. 